\title{
Dynamic Radiation Effects Induced by Short-Pulsed GeV U-Ion Beams in Graphite and h-BN Targets
}

\author{
Philipp Bolz (D), 1,2 Philipp Drechsel (D), ${ }^{1,2}$ Alexey Prosvetov ${ }^{1}$, ${ }^{1,2}$ Pascal Simon $\left(\mathbb{D},{ }^{1,2}\right.$ \\ Christina Trautmann $\left(\mathbb{D},{ }^{1,2}\right.$ and Marilena Tomut $\mathbb{D}^{1,3}$ \\ ${ }^{1}$ Materials Research Department, GSI Helmholtz Center for Heavy Ion Research, Planckstr 1, Darmstadt 64291, Germany \\ ${ }^{2}$ Department of Materials and Earth Sciences, Technische Universität Darmstadt, Alarich-Weiss-Straße 2, \\ Darmstadt 64287, Germany \\ ${ }^{3}$ Institute of Materials Physics, WWU Münster, Wilhelm-Klemm-Straße 10, Münster 48149, Germany
}

Correspondence should be addressed to Philipp Bolz; p.bolz@gsi.de and Marilena Tomut; mtomut@uni-muenster.de

Received 1 September 2020; Accepted 25 August 2021; Published 18 September 2021

Academic Editor: Mickael Lallart

Copyright (c) 2021 Philipp Bolz et al. This is an open access article distributed under the Creative Commons Attribution License, which permits unrestricted use, distribution, and reproduction in any medium, provided the original work is properly cited.

\begin{abstract}
Targets of isotropic graphite and hexagonal boron nitride were exposed to short pulses of uranium ions with $\sim 1 \mathrm{GeV}$ kinetic energy. The deposited power density of $\sim 3 \mathrm{MW} / \mathrm{cm}^{3}$ generates thermal stress in the samples leading to pressure waves. The velocity of the respective motion of the target surface was measured by laser Doppler vibrometry. The bending modes are identified as the dominant components in the velocity signal recorded as a function of time. With accumulated radiation damage, the bending mode frequency shifts towards higher values. Based on this shift, Young's modulus of irradiated isotropic graphite is determined by comparison with ANSYS simulations. The increase of Young's modulus up to 3 times the pristine value for the highest accumulated fluence of $3 \times 10^{13}$ ions $/ \mathrm{cm}^{2}$ is attributed to the beam-induced microstructural evolution into a disordered structure similar to glassy carbon. Young's modulus values deduced from microindentation measurements are similar, confirming the validity of the method. Beam-induced stress waves remain in the elastic regime, and no large-scale damage can be observed in graphite. Hexagonal boron nitride shows lower radiation resistance. Circular cracks are generated already at low fluences, risking material failure when applied in high-dose environment.
\end{abstract}

\section{Introduction}

With a new generation of particle accelerators like the Facility for Antiproton and Ion Research (FAIR) or the High Luminosity LHC at CERN, unprecedented high beam intensities will be reached. Under conditions of short pulse duration, the impact of intense ion beams on intercepting devices such as production targets [1], collimators [2], or beam dumps might result in failure of these components. Experiments measuring pulsed-beam-induced effects were previously conducted at the HiRadMat beamline at CERN [3-7], at Brookhaven National Laboratory [8], and at SIS18 at GSI [9]. This work presents results from irradiations with $\sim 1 \mathrm{GeV}$ uranium ions at the linear accelerator UNILAC at GSI Helmholtz Center for Heavy Ion Research in Darmstadt (Germany). The ion mass is higher, and the energy is lower than beams used in earlier studies [3-9]. The energy close to the Bragg peak provides maximum energy loss and thus leads to fast dose accumulation [10-12]. The lower energy has also the advantage of rather limited sample activation. Such beam conditions allow efficient monitoring of the response of the target materials under high-intensity shortpulse beams and as a function of radiation damage accumulation. Experiments with accelerated dose accumulation are important in order to test the suitability of production target or beam dump materials under long-term operation conditions.

In thin targets, beam-induced rapid heat deposition leads to bending and radial and transversal waves, which we observed by measuring the surface velocities using laser Doppler vibrometry. A critical parameter for the numerical modelling of these effects is Young's modulus. With 
increasing radiation damage, Young's modulus can change $[13,14]$. This leads to a shift of the frequency of beam-induced bending modes. Comparing experimental frequency data to results of ANSYS simulations allows in situ monitoring of the evolution of Young's modulus during irradiation. Independent offline microindentation measurements on a fluence series of irradiated samples were used to validate Young's modulus values deduced during the irradiation. The investigated materials are isotropic graphite and sintered boron nitride (h-BN). Due to its usage in nuclear reactors, radiation damage induced by neutrons and protons in graphite is well documented [15-18] while data of swift heavy ion induced damage are scarcer $[13,14]$. The boron nitride grade has a hexagonal structure similar to graphite, but its elastic modulus is larger and its thermal conductivity is lower. In a previous proton irradiation experiment, X-ray diffraction analysis showed a smaller shift and less broadening of the (002) reflection indicating a higher radiation hardness of h-BN compared to graphite [19]. In the past, both materials have been considered as candidates for beam dumps and production targets $[1,20]$ or as luminescence screens in accelerators. Investigating the response of the two materials contributes to a better understanding of how radiation damage affects their response to beam impact and which online monitoring techniques can be used to detect critical degradation of beam intercepting devices in an early stage and thus avoid catastrophic failures during beamtimes.

\section{Materials and Methods}

The isotropic graphite grade investigated was SIGRAFINE R6650 [21] produced by SGL Group with an average grain size of $7 \mu \mathrm{m}$. The disk-shaped sample (diameter of $20 \mathrm{~mm}$ ) was cut from a larger piece and lapped on both sides to a thickness of $0.5 \mathrm{~mm}$. The h-BN sample was isotropic HeBoSint D100 grade produced by Henze Boron Nitride Products [22] of the same size as the graphite specimen. Table 1 summarizes the properties of the materials [21-24]. Both materials have a similar mass density, Poisson ratio, coefficient of thermal expansion, and heat capacity, but h-BN has a $\sim 2$ times higher Young's modulus and a $\sim 4$ times lower thermal conductivity than graphite. For the same impacting beam intensity, higher amplitudes of thermal stress waves have to be expected.

The discs of isotropic graphite and h-BN were mounted in a ring-shaped sample holder as shown in Figure 1(a). The outer millimetre of the samples is clamped to the holder, resulting in a free-standing diameter of the disc of $18 \mathrm{~mm}$. The holders were mounted at the M3 beamline of the UNILAC using the setup depicted in Figure 1(b). The targets were exposed to $100 \mu \mathrm{s}$-long $\mathrm{U}$-ion pulses with a kinetic energy of $1.14 \mathrm{GeV}$ and a beam repetition rate of $0.5 \mathrm{~Hz}$. The beam was shaped with horizontal and vertical slits, resulting in a homogenous beam spot with a square shape and an area of $10 \times 10 \mathrm{~mm}^{2}$ positioned in the centre of the samples. Position, shape, and homogeneity of the beam were controlled with a luminescence screen mounted on the sample holder as shown in Figure 1(a). The energy loss at the surface and the beam penetration depth were calculated with the SRIM-2013 code
[25] (Figure 2). In Table 2, they are listed together with the beam parameters. Beam-induced heating of the samples was controlled with a thermal camera and kept below $200^{\circ} \mathrm{C}$. This secures that the properties of the target materials remain close to the values at room temperature given in the data sheets. The thermal camera was positioned normal to the beam axis observing at the same time the temperature on the front and back side of the sample with help of two infrared gold mirrors. The surface velocity related to the beam-induced bending and stress waves in the samples was measured with a Polytec OFV525/-5000-S laser Doppler vibrometer (LDV). The laser is directed to the sample and reflected from the surface. The frequency of the reflected light of a moving object is changed due to the Doppler effect. Comparing the frequency shift of the reflected light to the initial laser signal allowed us to determine the velocity of the sample surface and thus derive the frequency and amplitude of thermoelastic stress waves. The LDV was aligned at the rear side at $45^{\circ}$ angle to avoid any beam-induced damage to the LDV and the corresponding view port at the beamline. A tiny piece of adhesive reflective tape with an area of about $1 \mathrm{~mm}^{2}$ was glued at the centre of the sample to increase the reflectivity for the laser beam and to avoid the loss of the reflected laser beam during surface displacement. The LDV was connected to an oscilloscope allowing signal acquisition with a temporal resolution of $1 \mathrm{~ns}$. Before each LDV measurement, the beam was switched off to let the samples cool down to room temperature. Subsequently, the velocity signal generated by the first pulse, after switching on the beam again, was recorded.

To benchmark the results of the LDV measurements, simulations with ANSYS 2019 R2 release were conducted. A quarter of the disc was simulated using the properties given in Table 1. A schematic drawing of geometry and orientation is shown in Figure 3. The energy loss of the ions was simplified by depositing the total kinetic energy across the penetration depth resulting in a constant heat generation of $3 \mathrm{MW} / \mathrm{cm}^{3}$ in the irradiated volume for the pulse duration of $100 \mu \mathrm{s}$. To simulate the effects of beam-induced material degradation, simulations were also conducted for isotropic graphite with increased Young's moduli and reduced thermal conductivities [26] in the irradiated volume.

For indentation experiments, SIGRAFINE R6650 square samples of $10 \times 10 \mathrm{~mm}^{2}$ with a thickness of $2 \mathrm{~mm}$ were cut and lapped from the top. The samples were irradiated to different fluences with a flux of $1-3 \times 10^{9}$ ions $/ \mathrm{cm}^{2} \cdot s$. One half of each sample was masked with a thick aluminium foil which allowed a direct comparison of the irradiated part with the pristine part. Microindentation using a NanoTest Vantage system by Micro Materials with a Berkovich indenter tip was conducted on the irradiated samples up to a maximum indentation depth of $10 \mu \mathrm{m}$ (contact area larger than $1000 \mu \mathrm{m}^{2}$ ). This is large enough to penetrate several grains and at the same time small enough to remain in the region of constant electronic energy loss (Figure 2) and to avoid the influence of the pristine substrate. The unloading curves were analysed by the Oliver-Pharr method to obtain Young's modulus $[27,28]$. Measurements on at least 25 positions were taken on each sample both for the pristine part and the part exposed to the ion beam. 
TABLE 1: Properties of isotropic graphite grade SIGRAFINE R6650 produced by SGL Group and h-BN grade HeBoSint D100 produced by Henze Boron Nitride Products, used in this work.

\begin{tabular}{lcc}
\hline Property & Graphite SGL R6650 [21] & h-BN HeBoSint D100 [22] \\
\hline Density $\rho\left(\mathrm{g} / \mathrm{cm}^{3}\right)$ & 1.84 & 2.0 \\
Young's modulus $E(\mathrm{GPa})$ & 12.5 & 23 \\
Poisson's ratio $\nu$ & $0.19[23]$ & 4.1 \\
Coefficient of thermal expansion $\alpha\left(10^{-6} / \mathrm{K}\right)$ & 95 & 3.0 \\
Thermal conductivity $\lambda(\mathrm{W} / \mathrm{m} \cdot \mathrm{K})$ & 0.7 & 25 \\
Specific heat $c_{\mathrm{p}}(\mathrm{J} / \mathrm{g} \cdot \mathrm{K})$ & 0.6 \\
\hline
\end{tabular}

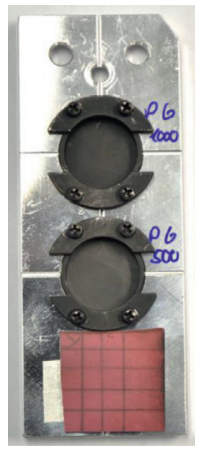

(a)

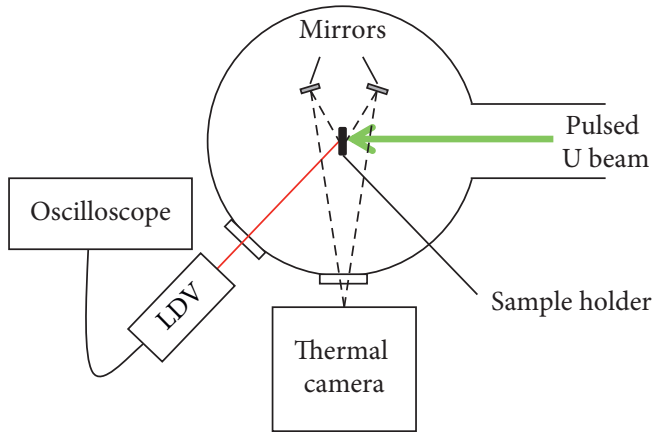

(b)

FIGURE 1: (a) Sample holder with two graphite samples mounted above a luminescence screen. (b) Schematic setup for online monitoring of elastic waves using a laser Doppler vibrometer and a thermal camera. The experiments were conducted at the M3 beamline at the UNILAC of GSI.

\section{Results and Discussion}

3.1. Isotropic Graphite Grade SIGRAFINE R6650. To estimate the maximum temperature rise per pulse, it is assumed that the entire power density of $3 \mathrm{MW} / \mathrm{cm}^{3}$ of a $\mathrm{U}$-ion pulse is transferred into heat. This value was inserted in the ANSYS simulations continuously over the duration of the $100 \mu \mathrm{s}$-long ion pulse. We simulated a quarter of a disc with a diameter of $20 \mathrm{~mm}$ and a thickness of $0.5 \mathrm{~mm}$ with adiabatic boundary conditions (justified by the short time scales of the involved processes). Figure 4(a) shows the simulated temperature evolution of a single beam pulse on an isotropic graphite disc at different depths and radial positions. The temperature of the quasipristine sample increases during the duration of the pulse and reaches a maximum of $156^{\circ} \mathrm{C}$ at the end of the ion pulse at $100 \mu \mathrm{s}$ in the centre of the beam spot $(y=0$, $z=0$ ). The generated heat is conducted through the sample equilibrating within $1 \mathrm{~ms}$ to almost similar temperature values on both surfaces of the disc $(y=0$, compare $z=0$ with $z=0.5)$. Over the entire time, the outer rim of the disc $(y=10, z=0)$ remains at room temperature, confirming that the use of adiabatic conditions in the investigated time frame is adequate.

To simulate the case of a radiation-damaged sample, the thermal conductivity of the volume exposed to the beam was reduced to $4 \mathrm{~W} / \mathrm{m} \cdot \mathrm{K}$ [26]. The density was kept constant since radiation-induced swelling was shown to be below $5 \%$ $[29,30]$. The values of specific heat and coefficient of thermal expansion are expected to change towards those of glassy carbon $[26,31]$. As the difference of both these properties is small between graphite and glassy carbon, they were kept constant in the simulation. Due to the degraded properties, the temperature at the sample surface $(y=0, z=0)$ increases to $240^{\circ} \mathrm{C}$ after a U-ion pulse of $100 \mu$ s duration. A temperature of $255^{\circ} \mathrm{C}$ is obtained if no heat conduction is included, illustrating the severely limited heat conduction from the front of the sample within the time duration of the pulse. The temperature in a depth where the ions stop $(y=0$, $z=0.06)$ is lower than observed for the pristine sample. For damaged material, the dissipation of the energy deposited in the central beam spot is obviously slowed down, whereas heat conduction into the pristine surrounding is not affected. After $1 \mathrm{~ms}$, the temperature difference between the front and the back surface of the sample, at the central spot of the beam $(y=0$, compare $z=0$ with $z=0.5)$, is still larger than $50^{\circ} \mathrm{C}$.

The generated heat in the beam spot leads to thermal expansion of the material. Since the heating is initially affecting only one surface of the disc, bending modes are generated. To understand the signal of the LDV, the expected first-order bending frequency $f_{b 1}$ of a fixed and a free circular plate is calculated using equation (1) [32]. The respective radius of the unclamped part $(0.9 \mathrm{~cm})$ or the free disc $(1 \mathrm{~cm})$ is $r$, the thickness is $t$, Young's modulus is $E$, the density is $\rho$, and Poisson's ratio is $\nu$. $\lambda$ is a constant depending on the binding condition and Poisson's ratio and corresponds to 3.196 for a fixed binding condition and 2.958 (graphite) and 2.970 (h-BN) for a free binding condition [32]. 


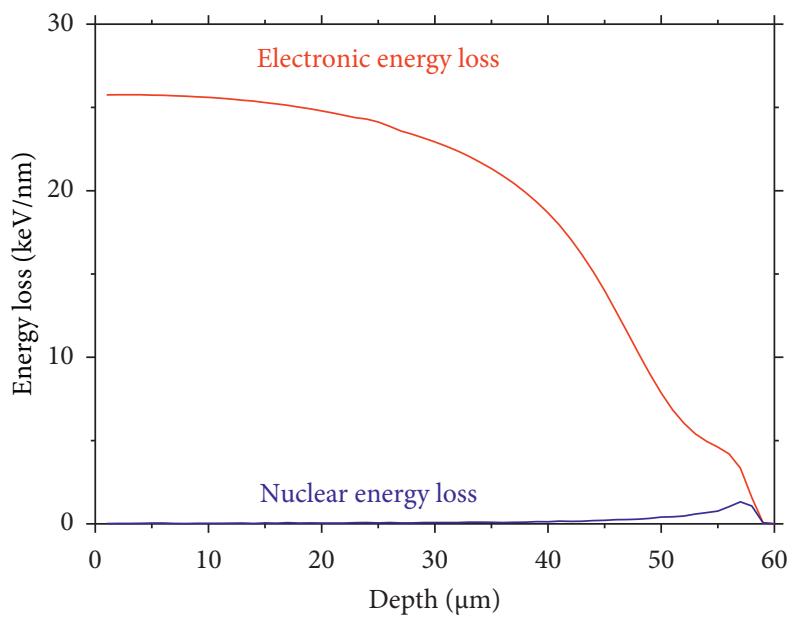

FIGURE 2: Electronic and nuclear energy loss as a function of penetration depth for $1.14 \mathrm{GeV}$ U-ions in a carbon target with a density of $1.84 \mathrm{~g} / \mathrm{cm}^{3}$ as calculated using the SRIM-2013 code.

TABLE 2: Penetration depth and surface energy loss as calculated with the SRIM-2013 code [25] and beam parameters of isotropic graphite grade SIGRAFINE R6650 and h-BN grade HeBoSint D100.

\begin{tabular}{lcc}
\hline & SGL R6650 & HeBoSint D100 \\
\hline Penetration depth $(\mu \mathrm{m})$ & 58 & 56 \\
Energy loss at surface $(\mathrm{keV} / \mathrm{nm})$ & 25.7 & 26.8 \\
Pulse length $(\mu \mathrm{s})$ & 100 & 100 \\
Intensity (ions $/ \mathrm{cm}^{2} \cdot$ pulse) & $1.0-1.6 \times 10^{10}$ & $1.0-1.6 \times 10^{10}$ \\
Achieved fluence $\left(\right.$ ions $\left./ \mathrm{cm}^{2}\right)$ & $3.5 \times 10^{13}$ & $5 \times 10^{13}$ \\
\hline
\end{tabular}

$$
f_{b 1}=\frac{\lambda^{2}}{2 \pi \cdot r^{2}} \cdot \sqrt{\frac{E \cdot t^{2}}{12 \cdot \rho \cdot\left(1-\nu^{2}\right)}}
$$

Due to the short time of the ion pulse, the thermal inertia of the outer material prevents the expansion of the heated beam spot, causing thermal stress. This stress generates stress waves which travel through the sample and are reflected at the interfaces. The frequency of the transversal elastic wave $f_{t r}$, which is reflected between the back and front surfaces of the sample, is calculated using the following equation [33]:

$$
f_{\mathrm{tr}}=\frac{1}{2 \cdot t} \cdot \sqrt{\frac{E \cdot(1-\nu)}{\rho \cdot(1+\nu) \cdot(1-2 \nu)}} .
$$

The calculated frequencies for the investigated materials are summarized in Table 3. The frequencies expected for the transversal stress waves are three orders of magnitude larger, allowing an easy differentiation from the bending frequencies.

Figures 5(a) and 5(b) show velocity signals and corresponding fast Fourier transform (FFT) spectra measured with the LDV at the rear side $(x=y \approx 0, z=0.5)$ of a $500 \mu \mathrm{m}$ thick SGL R6650 disc impacted with $1.14 \mathrm{GeV}$ U-ions. For clarity, only data for the quasipristine sample as well as after the accumulation of a fluence of $1 \times 10^{13}$ and $3.5 \times 10^{13}$ ions $/ \mathrm{cm}^{2}$ are presented. Due to the alignment of the LDV under an angle of $45^{\circ}$, the measured amplitude is significantly reduced and proper transformation from the voltage signal to velocity is difficult. Moreover, the amplitude slightly fluctuates from pulse to pulse due to beam intensity variations. Thus, the raw voltage signal is presented and focus is put on the frequency spectra of the involved processes. In the pristine sample, a harmonic oscillation with a frequency of $5.38 \pm 0.02 \mathrm{kHz}$ is generated in excellent agreement with the frequency $f_{b 1 \text {, free }}$ calculated for the free binding condition (Table 3 ). With increasing U-ion fluence, the frequency signal increases to $6.10 \pm 0.02 \mathrm{kHz}$ at the maximum applied fluence of $3.5 \times 10^{13} \mathrm{ions} / \mathrm{cm}^{2}$. We ascribe the increase of the frequency to an increase of Young's modulus in the irradiated area, assuming that the boundary conditions remained constant. Neither swelling was detected by means of profilometry nor macroscopic failures of the samples such as cracks were observed using optical microscopy. Irradiation of graphite results in the formation of interstitials and vacancies. Agglomeration of these defects creates vacancy clusters [34]. These defects prevent movement of dislocations and sliding of basal planes, causing a significant hardening and increase of Young's modulus.

ANSYS simulations of the deformation of the graphite disc were performed to infer beam-induced changes of Young's modulus. In a first step, the thermal input of Figure 4(a) was used on a pristine disc. The velocity and the corresponding FFT spectrum in the centre of the rear side $(x=y=0, z=0.5)$ of the disc with free boundary conditions are shown in Figures 5(c) and 5(d). An oscillation with a frequency of $5.32 \pm 0.02 \mathrm{kHz}$ is obtained, similar to the calculated value using equation (1) and measured with the LDV on the pristine sample. 


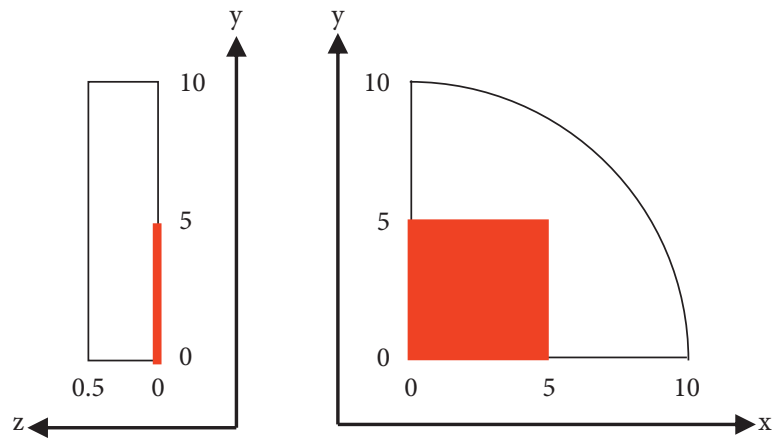

FIGURE 3: Schematic drawing in side and front views of a quarter of the simulated target disc with the plane of the disc oriented in $x$ - and $y$ directions and the thickness in $z$-direction. All units are in $\mathrm{mm}$. Note that the $z$-direction is not drawn to scale for better view. The beam spot is marked in red. The velocity is measured in the centre of the rear side $(x=y \approx 0, z=0.5)$.

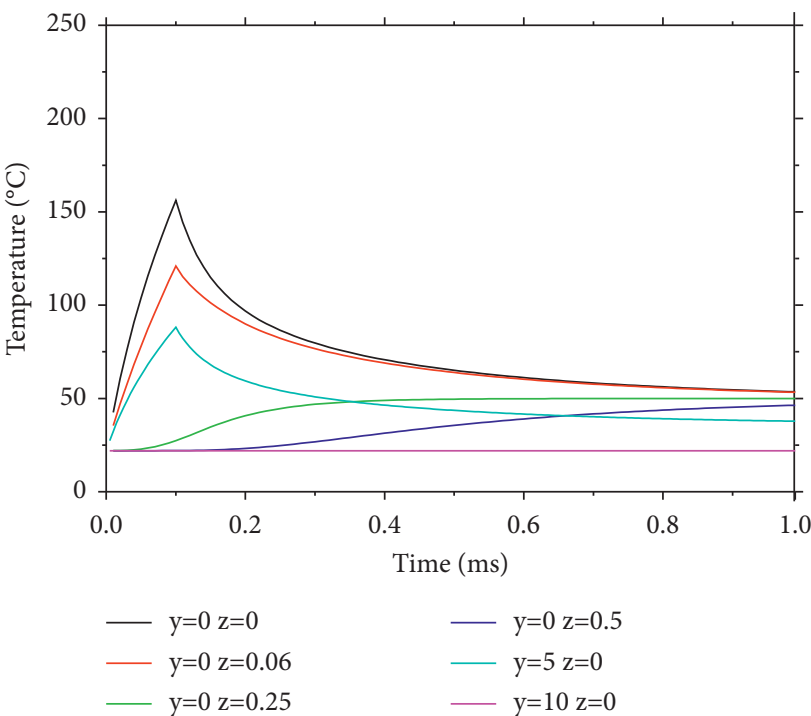

(a)

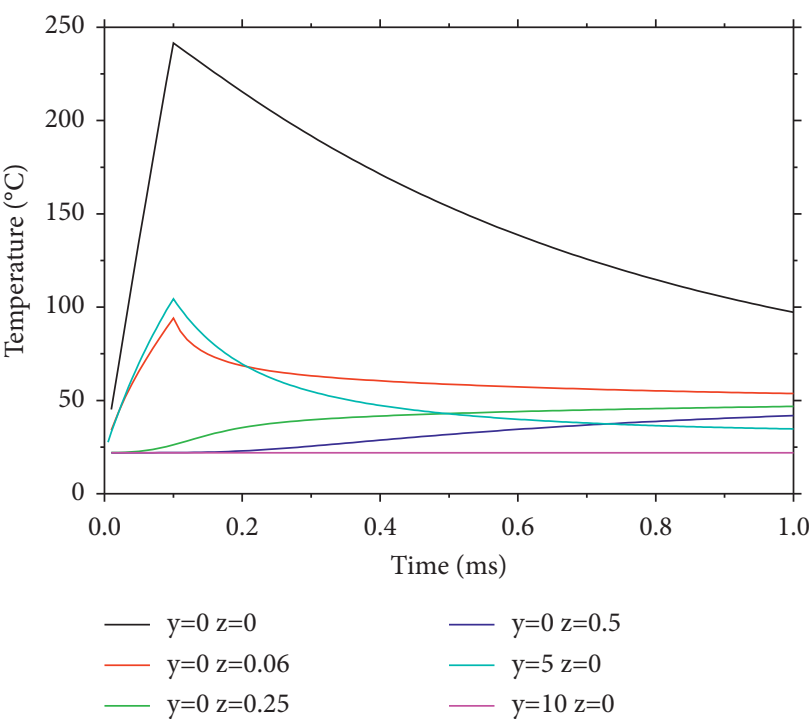

(b)

FIGURE 4: ANSYS-simulated time-dependent temperature evolution of a $0.5 \mathrm{~mm}$-thick isotropic graphite disc for $1 \mathrm{~ms}$ after the impact of a $100 \mu$ s-long U-ion pulse. Coordinates can be seen in Figure 3, with all points being at $x=0$. (a) Pristine material. (b) Thermal conductivity reduced to $4 \mathrm{~W} / \mathrm{m} \cdot \mathrm{K}$ in the material of the beam spot representing graphite after $1.14 \mathrm{GeV} \mathrm{U}$-ion irradiation to a fluence of $3 \times 10^{13}$ ions $/ \mathrm{cm}^{2}$ [26].

In a second step, ANSYS simulations of discs with larger Young's modulus in the beam spot were conducted. In Figures 5(c) and 5(d), examples of the velocity in the centre of the rear side of discs with 2 and 3 times the pristine Young's modulus are shown. The properties of the part outside the beam spot were assumed to stay constant since the temperature increase is not high enough to significantly change the properties (as seen in Figure 4). The frequency spectra of the LDV recordings at different fluences can now be compared with the frequency results of the ANSYS simulations as demonstrated in Figure 6(a) for 2 and 3 times the pristine Young's modulus. The frequencies for those two simulations were measured in the LDV signal at accumulated fluences of $1.5 \times 10^{13}$ and $3.3 \times 10^{13}$ ions $/ \mathrm{cm}^{2}$, respectively. The same procedure was used to estimate a continuous development of Young's modulus depending on the fluence as shown in Figure 6(b).
To test the validity of this method, the deduced Young's modulus values are compared to microindentation measurements on a sample series with different accumulated fluences. From the good agreement in the investigated fluence range, it can be concluded that the LDV online measurement method provides realistic results for beaminduced changes of Young's modulus for isotropic graphite. This is excellent news because it reduces the need to irradiate samples series with different accumulated fluences and avoids uncertainties due to property fluctuations between several samples.

Both methods show a large increase up to about 3 times the pristine Young's modulus $E_{0}$ for irradiation with a fluence of $3 \times 10^{13} \mathrm{U}$-ions $/ \mathrm{cm}^{2}$. The obtained increase is similar to the ratio of Young's moduli of glassy carbon [31] and SIGRAFINE R6650 [21] of 2.8. This supports the assumption that intensive ion irradiation induces a transition towards a disordered 
TABLE 3: Calculated first-order frequencies for bending and transversal elastic stress waves of isotropic graphite grade SIGRAFINE R6650 and h-BN grade HeBoSint D100.

\begin{tabular}{lcc}
\hline & SGL R6650 & HeBoSint D100 \\
\hline$f_{b 1, \text { fixed }}(\mathrm{kHz})$ & 7.69 & 10.07 \\
$f_{b 1, \text { free }}(\mathrm{kHz})$ & 5.32 & 7.04 \\
$f_{t r}(\mathrm{MHz})$ & 2.73 & 3.62 \\
\hline
\end{tabular}

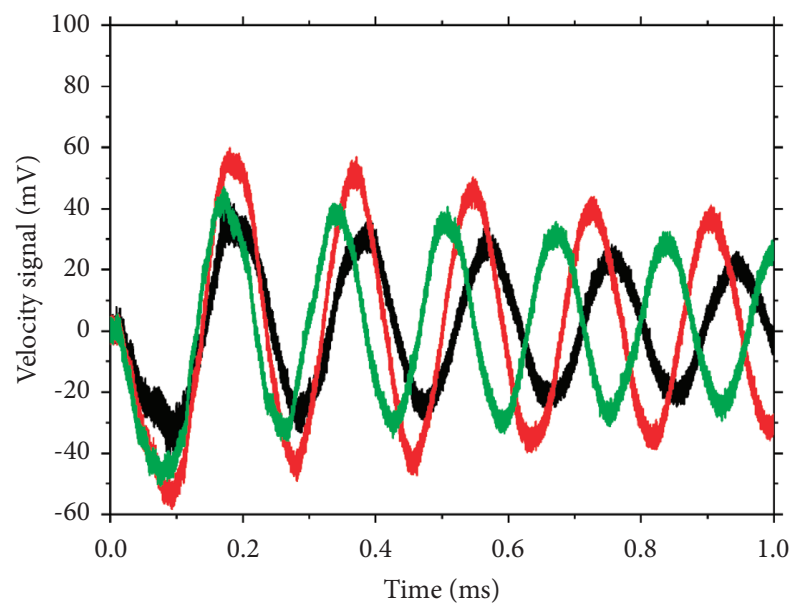

- Pristine
$-\quad 1 \mathrm{e} 13 \mathrm{U}$ ions $/ \mathrm{cm}^{2}$
$-\quad 3.5 \mathrm{e} 13 \mathrm{U}$ ions $/ \mathrm{cm}^{2}$

(a)

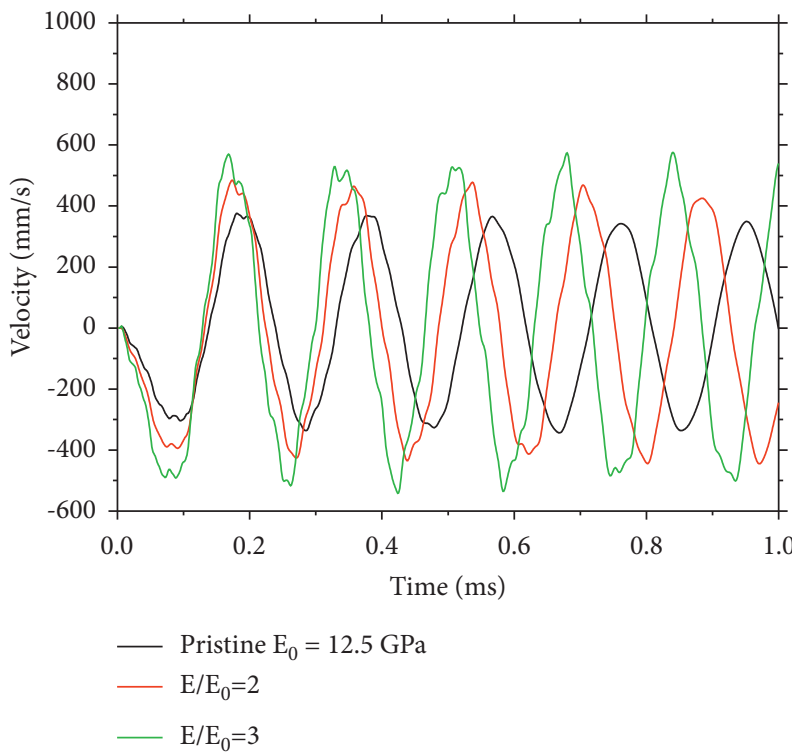

(c)

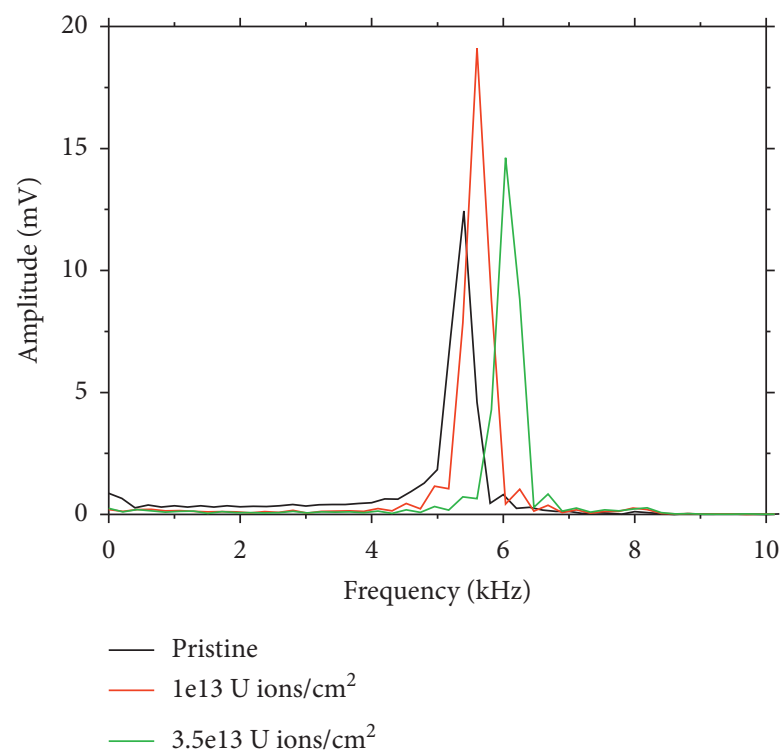

(b)

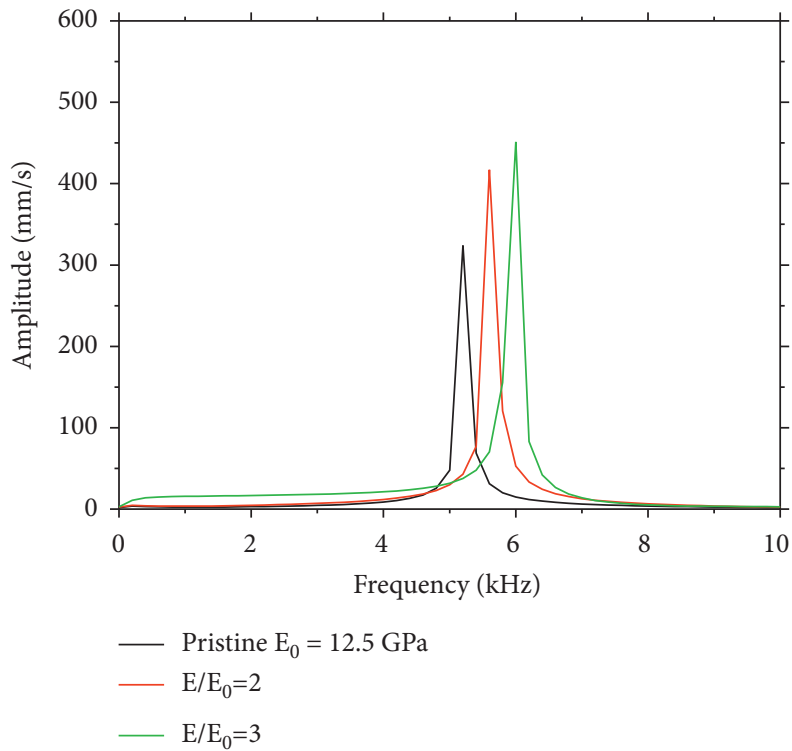

(d)

FigURE 5: (a) Time-dependent velocity signal measured by LDV and (b) corresponding FFT spectra at the rear side $(x=y \approx 0, z=0.5)$ of a free $0.5 \mathrm{~mm}$-thick isotropic SGL R6650 graphite disc for the irradiation with $1.14 \mathrm{GeV}$ U-ions at different fluences. Corresponding ANSYS simulations at $x=y=0, z=0.5$ : (c) time-dependent velocity and (d) FFT spectra for the impact of a $100 \mu$ s U-ion pulse (power density: $3 \mathrm{MW} / \mathrm{cm}^{3}$ ). Depicted are simulations of a pristine sample and samples with values of Young modulus 2 and 3 times higher than the pristine value as well as thermal conductivity degradation from $95 \mathrm{~W} / \mathrm{m} \cdot \mathrm{K}$ (pristine graphite) to $11 \mathrm{~W} / \mathrm{m} \cdot \mathrm{K}$ and $4 \mathrm{~W} / \mathrm{m} \cdot \mathrm{K}$, respectively [26], in the heated volume. 


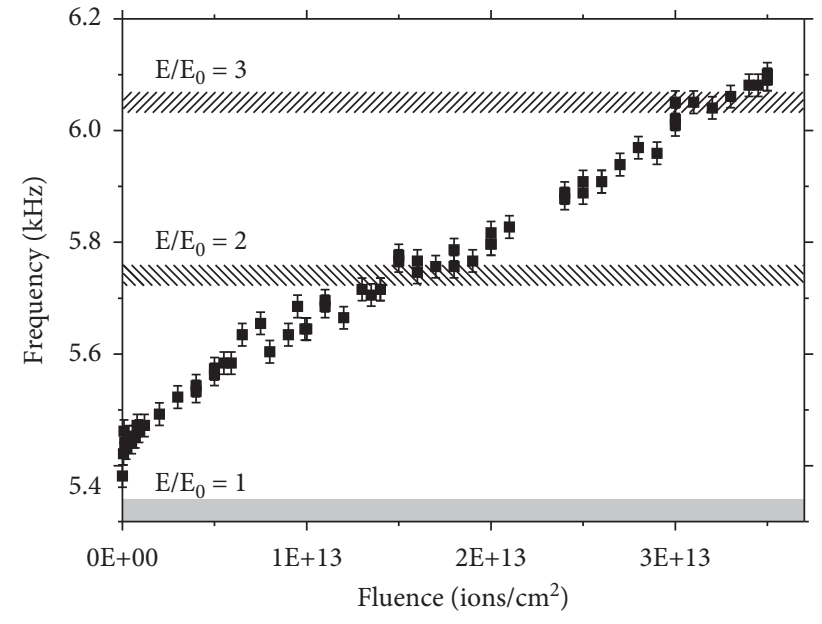

(a)

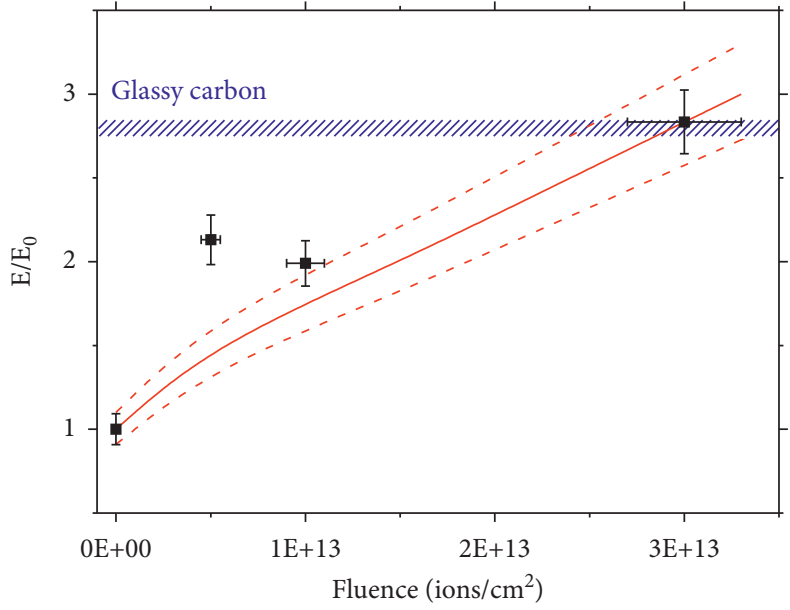

— Frequency shift

- Microinentation

FIGURE 6: (a) Measured bending frequency of isotropic SGL R6650 graphite as a function of $1.14 \mathrm{GeV}$ U-ion accumulated fluence. Shaded areas represent the frequencies obtained by ANSYS simulations with pristine properties as well as with 2 and 3 times the pristine Young's modulus in the beam spot. The uncertainty of the frequencies of $0.02 \mathrm{kHz}$ is obtained by the frequency resolution of the FFT. (b) Relative increase of Young's modulus as a function of the U-ion fluence measured by microindentation and deduced from simulations of frequency shifts of LDV velocity signals. Error bars represent the error of the mean value for at least 25 microindentation measurements. The uncertainty of the fluence and of Young's modulus determined from the frequency shift is estimated to be 10\% (red dashed lines). The shaded blue area represents the ratio of Young's modulus of the glassy carbon grade HTW SIGRADUR K [31] and SIGRAFINE R6650 [21] taken from the respective data sheets.

structure similar to glassy carbon $[14,26]$. Compared to neutron irradiation, the effects of heavy ion irradiation are much larger. The fluences achieved in this study correspond to less than $0.01 \mathrm{dpa}$ as calculated by SRIM Kinchin and Pease damage calculation $[25,35]$. Several tens of dpa are necessary for similar changes induced by neutron irradiation [15-17].

Besides the large amplitude caused by the bending of the sample, also elastic or plastic transversal stress waves are expected at a pulse length of $100 \mu$ s and a deposited power of approximately $3 \mathrm{MW} / \mathrm{cm}^{3}$ [36]. These transversal waves propagate along the disk axis and, according to the values calculated in Table 3, appear in the $\mathrm{MHz}$ frequency regime. Time-resolved frequency analysis of the LDV signal was performed by continuous wavelet transformation with a Morlet wavelet with a wave number of 20 . The results for the first dynamic response obtained for a pristine isotropic graphite target are shown in Figure 7. The highest wavelet coefficient appears at a frequency of $2.70 \pm 0.02 \mathrm{MHz}$, very close to the calculated frequency of $2.73 \mathrm{MHz}$. This signal corresponds to an elastic stress wave as the pressure wave propagates at the speed of sound [37]. The yield strength of graphite is not reached and no plastic deformation occurs. This supports our hypothesis that the sample properties outside the beam spot are not changed as inserted in the ANSYS simulations. The maximum of the wavelet coefficient is obtained at the end of the pulse of $100 \mu \mathrm{s}$. At this point, the highest values of temperature and velocity are reached, resulting in the highest thermal stress. After the end of the beam pulse, the temperature difference decreases and the stress wave signal is rapidly damped.
3.2. Hexagonal Boron Nitride. Isotropic hexagonal $\mathrm{BN}$ has a similar structure as graphite. However, its polar bonding results in a lower resistance to crack propagation. LDV velocity signals and corresponding FFT spectra measured at the rear side $(x=y \approx 0, z=0.5)$ of the sample are shown in Figure 8. At a low fluence of $4 \times 10^{11}$ ions $/ \mathrm{cm}^{2}$, a vibration with a main frequency of $9.53 \pm 0.07 \mathrm{kHz}$ was measured. According to the calculation with equation (1), $10.07 \mathrm{kHz}$ corresponds to a bending mode for a fixed sample. ANSYS simulations with a fixed support at the outer $1 \mathrm{~mm}$ rim show good agreement of the waveform with the measured velocity signals as presented in Figure 9.

Figure 10 shows the development of the bending frequency as a function of fluence in the frequency range around $10 \mathrm{kHz}$ (Figure 10(a)) and $40 \mathrm{kHz}$ (Figure 10(b)). The low-frequency signal remains rather stable at about $9.5 \mathrm{kHz}$ up to $2 \times 10^{12}$ ions $/ \mathrm{cm}^{2}$ and then increases to $10.6 \mathrm{kHz}$ for $1 \times 10^{13}$ ions $/ \mathrm{cm}^{2}$. For higher fluences, the signal fluctuates irregularly.

In the high-frequency regime, a new double-peak signal appears at a fluence of $2 \times 10^{12}$ ions $/ \mathrm{cm}^{2}$ with centres at $38.4 \pm 0.1$ and $39.4 \pm 0.1 \mathrm{kHz}$ (Figure $8(\mathrm{~b})$ ). These frequencies remain stable up to a fluence of $1 \times 10^{13} \mathrm{ions} / \mathrm{cm}^{2}$. With further fluence increase (Figure 10(b)), a fluctuation of highfrequency peaks in the FFT spectra similar to those in the low frequency regime occurs. We ascribe these frequency effects to the formation of cracks $\left(>2 \times 10^{12}\right.$ ions $\left./ \mathrm{cm}^{2}\right)$ and finally to the complete detachment of the beam-exposed central part of the sample $\left(>1 \times 10^{13}\right.$ ions $\left./ \mathrm{cm}^{2}\right)$. 


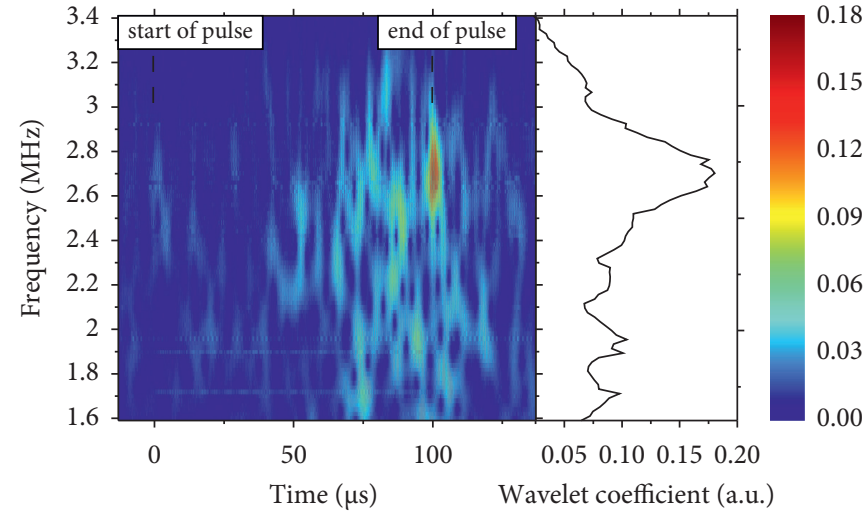

(a)

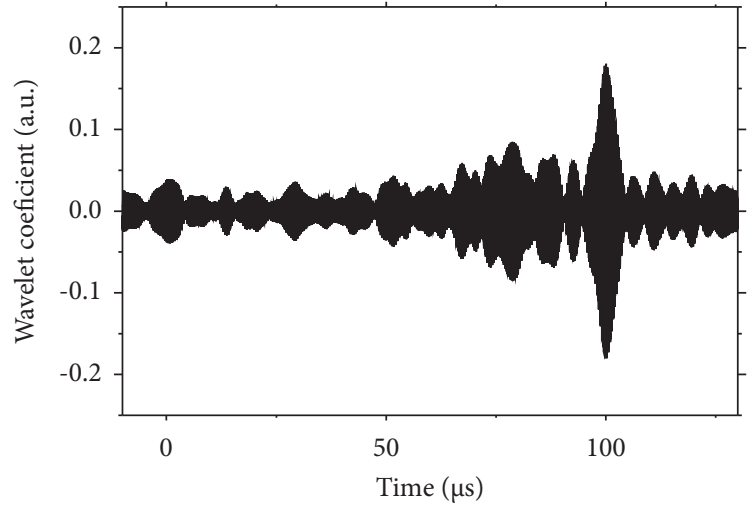

(b)

Figure 7: (a) Contour plot of the absolute wavelet coefficient as a function of time and frequency and maximum wavelet coefficient depending on the frequency. (b) Time-dependent wavelet coefficient at $2.70 \mathrm{MHz}$. Both graphs show the impact of the first $1.14 \mathrm{GeV} \mathrm{U}$-ion pulse on pristine isotropic graphite.

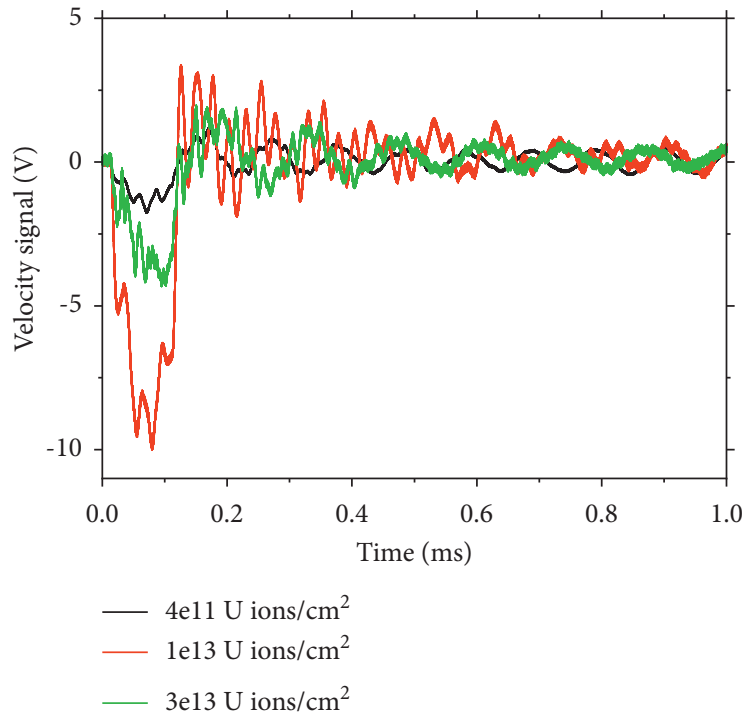

(a)

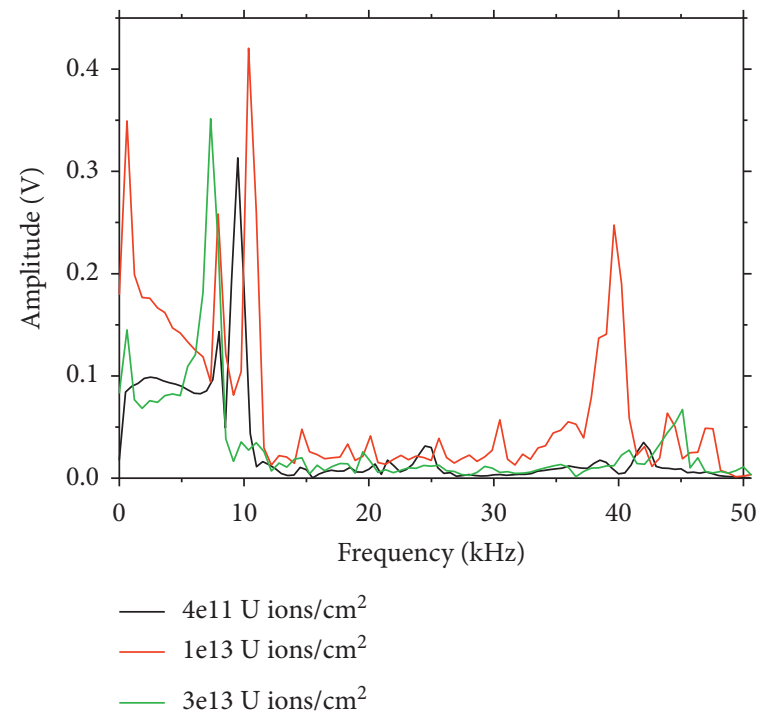

(b)

FIGURE 8: (a) LDV velocity signal as a function of time and (b) corresponding FFT spectra measured at the rear side $(x=y \approx 0, z=0.5)$ of a $0.5 \mathrm{~mm}$-thick h-BN disc under irradiation with $1.14 \mathrm{GeV} \mathrm{U}$-ions at different fluences.

Our assumption is supported by the observation during dismounting of the irradiated sample, where we identified a round piece of $8-9 \mathrm{~mm}$ in diameter completely detached from the rest of the sample (Figure 11). The fracture piece shows pronounced bending which is probably due to beaminduced swelling. Based on this one sample, quantitative evaluation of this effect was unfortunately not possible.

By using equation (1) for a sample diameter of $8.5 \mathrm{~mm}$, which is the average diameter of the detached central part of the h-BN target, a free bending frequency of $39.0 \mathrm{kHz}$ is calculated, fitting well the measured frequencies. The appearance of two peaks is ascribed to the noncircular shape of the detached piece. ANSYS simulations indicate that a Young's modulus of approximately 4 times the pristine value is required in the beam spot to reproduce the experimentally observed frequency changes at a fluence of $1 \times 10^{13}$ ions $/ \mathrm{cm}^{2}$. For a detached sample piece, this should result in even larger changes of the frequency within the beam spot which is not the case (Figure 10(b)). This indicates that the increase of the low frequency is primarily caused by cracks in the sample and not by an increase of Young's modulus.

In contrast to isotropic graphite, visible degradation of irradiated h-BN appears at smaller fluences, indicating a lower radiation resistance. While the graphitic samples kept their shape and integrity, h-BN shows a visible deformation which eventually results in cracking and fragmentation of the structure. First failure signs are visible in the velocity and FFT signals at fluences as low as $2 \times 10^{12}$ ions $/ \mathrm{cm}^{2}$. The damage effect becomes more pronounced at higher fluences 


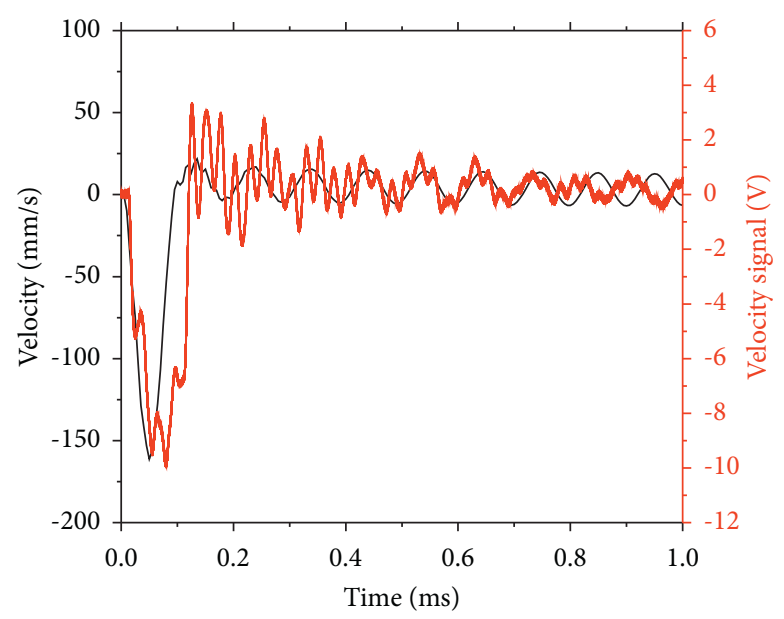

- ANSYS simulation

— LDV measurement

FIGURE 9: Time-dependent velocity signal at the rear side $(x=y \approx 0, z=0.5)$ of a $0.5 \mathrm{~mm}$-thick $\mathrm{h}$-BN disc from LDV measurements at an accumulated fluence of $1 \times 10^{13}$ ions $/ \mathrm{cm}^{2}$ (red) and simulated with ANSYS using pristine properties (black).

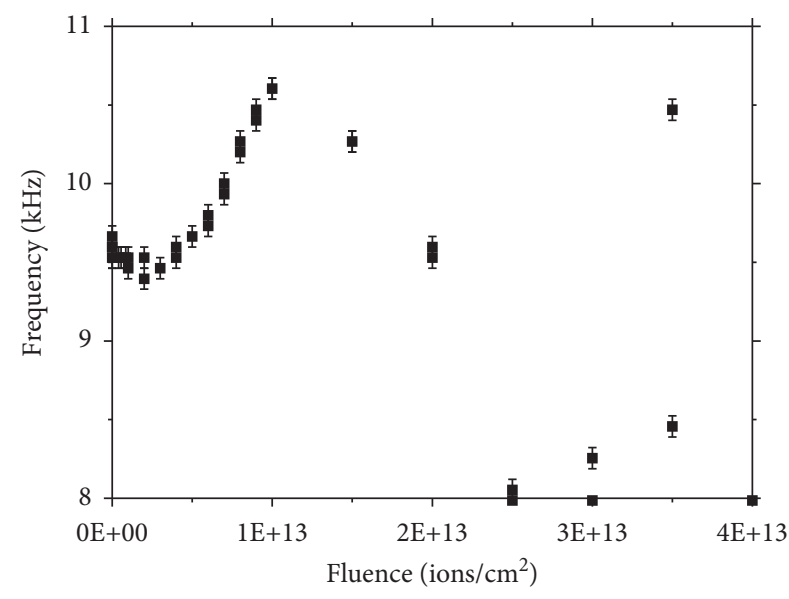

(a)

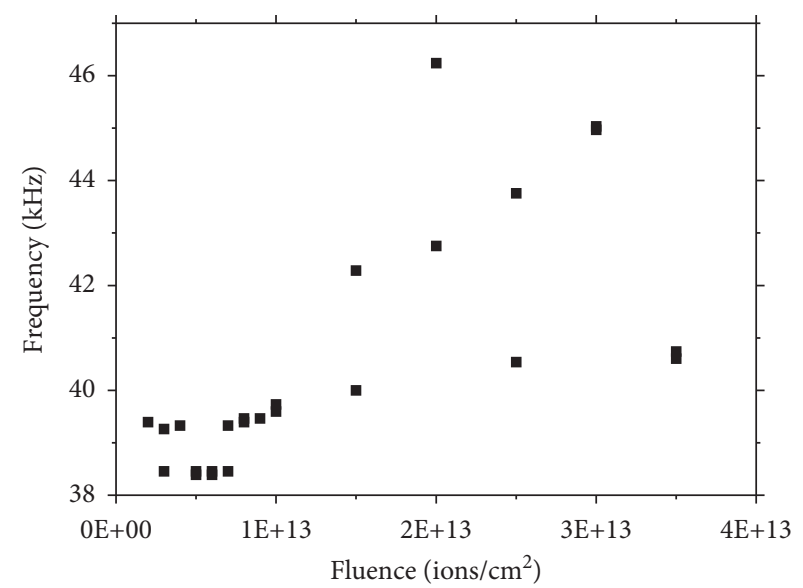

(b)

Figure 10: Measured frequency of the bending mode as a function of fluence for h-BN irradiated with $1.14 \mathrm{GeV}$ U-ions: (a) low-frequency signal around $10 \mathrm{kHz}$ and (b) high-frequency signal around $40 \mathrm{kHz}$. The large signal fluctuations above $1 \times 10^{13}$ ions $/ \mathrm{cm}^{2}$ are ascribed to fragmentation/detachment of the irradiated inner section of the sample. The uncertainty of the frequencies is due to the limited frequency resolution of the FFT.

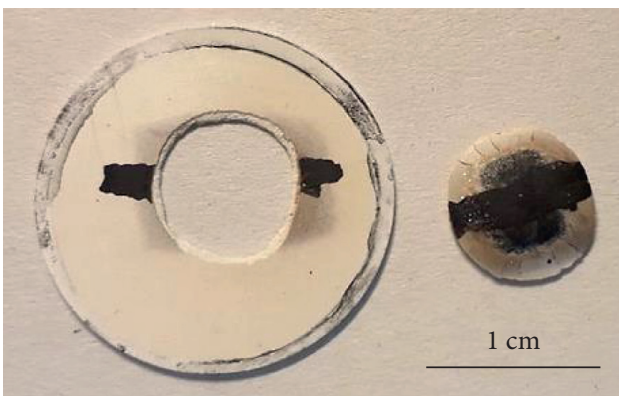

FIGURE 11: h-BN sample after irradiation with $1.14 \mathrm{GeV}$ U-ions to an accumulated fluence of $5 \times 10^{13}$ ions $/ \mathrm{cm}^{2}$. The sample fractured around the beam spot area and is visibly bent. The beam darkened the sample. The black horizontal line originates from a carbon paint applied for thermal camera monitoring during irradiation. 
as shown for the signal at a fluence of $1 \times 10^{13}$ ions $/ \mathrm{cm}^{2}$. While h-BN was demonstrated to be stable under continuous proton irradiation [19], the larger energy loss and the pulsed structure of the $\mathrm{U}$-ion beam result in stress concentration profiles appearing at the interface between irradiated and pristine material leading to crack formation. Due to the brittle nature of $\mathrm{h}-\mathrm{BN}$, the irradiated part of the target finally detaches from the sample even though the penetration depth of the ion beam is only about one tenth of the sample thickness. In contrast, graphite is characterized by slow crack growth and self-healing of preexisting microcracks due to growth in the $c$-axis direction [38, 39], leading to higher critical stress values. Moreover, in irradiated graphite, stress is accommodated by creep deformation preventing catastrophic failures.

\section{Conclusions}

Discs of isotropic graphite and hexagonal boron nitride were exposed to $\mathrm{U}$-ion pulses with $\mathrm{GeV}$ kinetic energy. The dynamic response of the targets was monitored by recording the surface velocity signal at the rear side using laser Doppler vibrometry. Beam-induced changes of the first-order bending frequency of the isotropic graphite disc allow online determination of Young's modulus of irradiated material by comparison with ANSYS simulations. Young's modulus rises to approximately 3 times the pristine value for a fluence of $3.5 \times 10^{13} \mathrm{U}$-ions $/ \mathrm{cm}^{2}$. The comparison with microindentation data shows good agreement, suggesting the online method as an interesting alternative to offline measurements of a series of samples that have been irradiated at different fluences. The observation by continuous wavelet transformation enables the detection of transversal elastic stress waves for the investigated pulse intensities of up to $1.6 \times 10^{10}$ ions $/ \mathrm{cm}^{2}$ per pulse. In the case of $\mathrm{h}-\mathrm{BN}$, cracks formed around the beam spot at a fluence of $2 \times 10^{12}$ ions $/ \mathrm{cm}^{2}$ which eventually result in failure of the disc. Our observations provide clear evidence that graphite exhibits a higher radiation hardness making it superior for applications in targets and beam dumps for high power accelerators. To provide safe operation conditions and estimate the life time of beam intercepting devices, the large increase of Young's modulus in graphite needs to be taken into account when simulating beam conditions and designing components. Our study also demonstrates that laser Doppler vibrometry is a suitable tool to monitor failures of beam-exposed devices by early detection of defect formation in materials, resulting in shifting frequencies or appearance of new frequency peaks, before catastrophic failures happen.

\section{Data Availability}

The experimental and simulation data used to support the findings of this study are available from the corresponding author upon request.

\section{Disclosure}

The results presented here are based on a UMAT experiment, which was performed at the M-branch of the UNILAC at the
GSI Helmholtzzentrum für Schwerionenforschung, Darmstadt (Germany), in the frame of FAIR Phase-0.

\section{Conflicts of Interest}

The authors declare that there are no conflicts of interest regarding the publication of this paper.

\section{Acknowledgments}

This study was funded by the European Union's Horizon 2020 Research and Innovation Program under grant agreement no. 730871. PB gratefully acknowledges the support from the HGS-HIRe Graduate School.

\section{References}

[1] H. Geissel, M. Winkler, H. Weick et al., "Technical design report on the super-FRS," Available from: http://repository. gsi.de/record/54552, 2008.

[2] N. A. Tahir, R. Schmidt, M. Brugger et al., "Simulations of full impact of the large hadron collider beam with a solid graphite target," Laser and Particle Beams, vol. 27, no. 3, pp. 475-483, 2009.

[3] M. Pasquali, A. Bertarelli, C. Accettura et al., "Dynamic response of advanced materials impacted by particle beams: the multimat experiment," Journal of Dynamic Behavior of Materials, vol. 5, no. 3, pp. 266-295, 2019.

[4] A. Bertarelli, E. Berthomé, C. Carra et al., "Dynamic testing and characterization of advanced materials in a new experiment at CERN HiRadMat facility," Journal of Physics: Conference Series, vol. 8, Article ID 082021, 2018.

[5] A. Bertarelli, Analytical Study of Axisymmetric Transient Thermal Stresses in Graphite Target Rods for the CNGS Facility, Vol. EDMS383454, CERN EST-ME Technical Note 2003-005, Geneva, Switzerland, 2003.

[6] F. Carra, A. Dallocchio, A. Bertarelli, and L. Gentini, "Mechanical engineering and design of novel collimators for HLLHC," in Proceedings of the International Particle Accelerator Conference 2014, Dresden, Germany, June 2014.

[7] P. Simon, P. Drechsel, P. Katrik, K.-O. Voss, and P. Bolz, "Dynamic response of graphitic targets with tantalum cores impacted by pulsed $440-\mathrm{GeV}$ proton beams," Manuscript submitted for publication, 2020.

[8] N. Simos, H. Kirk, S. Kahn et al., "Study of graphite targets interacting with the 24 gev proton beam of the BNL muon target experiment," in Proceedings of the EPAC, pp. 27092711, Paris, France, May 2012.

[9] R. Wilfinger, A. Kelic, B. Achenbach, K. H. Behr, A. Bruenle, and R. Catherall, "Study of thermal stress waves induced by relativistic heavy-ion beams," GSI Scientific Report, vol. 2008, p. 75, 2007.

[10] K. Kupka, A. A. Leino, W. Ren et al., "Graphitization of amorphous carbon by swift heavy ion impacts: molecular dynamics simulation," Diamond and Related Materials, vol. 83, pp. 134-140, 2018.

[11] J. Habainy, Y. Lee, K. B. Surreddi et al., "Study of heavy ion beam induced damage in tungsten for high power target applications," Nuclear Instruments and Methods in Physics Research Section B: Beam Interactions with Materials and Atoms, vol. 439, pp. 7-16, 2019.

[12] S. Fernandes, F. Pellemoine, M. Tomut et al., "In-situ electric resistance measurements and annealing effects of graphite 
exposed to swift heavy ions," Nuclear Instruments and Methods in Physics Research Section B: Beam Interactions with Materials and Atoms, vol. 314, pp. 125-129, 2013.

[13] C. Hubert, K. O. Voss, M. Bender et al., "Swift heavy ioninduced radiation damage in isotropic graphite studied by micro-indentation and in-situ electrical resistivity," Nuclear Instruments and Methods in Physics Research Section B: Beam Interactions with Materials and Atoms, vol. 365, pp. 509-514, 2015.

[14] I. Manika, J. Maniks, R. Zabels et al., "Nanoindentation and Raman spectroscopic study of graphite irradiated with swift $238 \mathrm{U}$ ions," Fullerenes, Nanotubes, and Carbon Nanostructures, vol. 20, no. 4-7, pp. 548-552, 2012.

[15] M. R. Bradford and A. G. Steer, "A structurally-based model of irradiated graphite properties," Journal of Nuclear Materials, vol. 381, no. 1-2, pp. 137-144, 2008.

[16] T. D. Burchell and W. P. Eatherly, "The effects of radiation damage on the properties of GraphNOL N3M," Journal of Nuclear Materials, vol. 179-181, pp. 205-208, 1991.

[17] G. Haag, "Properties of ATR-2E graphite and property changes due to fast neutron irradiation," Berichte des Forschungszentrums Julich, vol. 4183, 2005.

[18] N. Simos, P. Hurh, N. Mokhov et al., "Low-temperature proton irradiation damage of isotropic nuclear grade IG-430 graphite," Journal of Nuclear Materials, vol. 542, Article ID 152438, 2020.

[19] N. Simos, Z. Kotsina, D. Sprouster, Z. Zhong, H. Zhong, and P. Hurh, "Hexagonal boron nitride (h-BN) irradiated with 140 $\mathrm{MeV}$ protons," Nuclear Instruments and Methods in Physics Research Section B: Beam Interactions with Materials and Atoms, vol. 479, pp. 110-119, 2020.

[20] L. R. Evans, The Large Hadron ColliderA Marvel of Technology, EPFL Press, Lausanne, Switzerland, 1st edition, 2009.

[21] SGL Group, "SIGRAFINE R6650 data sheet," Available from: https://www.sglcarbon.com/pdf/SGL-DatenblattSIGRAFINE-R6650-DE.pdf (Accessed June 3, 2020), 2020.

[22] Henze Boron Nitride Products, "HeBoSint data sheet," Available from: https://www.henze-bnp.de/PDF/HeBoSint_ PD_D.pdf (Accessed May 4, 2017), 2017.

[23] A. Politano and G. Chiarello, "Probing the young's modulus and Poisson's ratio in graphene/metal interfaces and graphite: a comparative study," Nano Research, vol. 8, no. 6, pp. 1847-1856, 2015.

[24] Q. Peng, W. Ji, and S. De, "Mechanical properties of the hexagonal boron nitride monolayer: ab initio study," Computational Materials Science, vol. 56, pp. 11-17, 2012.

[25] J. Ziegler, M. Ziegler, and J. Biersack, "SRIM-the stopping and range of ions in matter," Nuclear Instruments and Methods in Physics Research B, vol. 268, no. 11-12, pp. 1818-1823, 2010.

[26] A. Prosvetov, G. Hamaoui, N. Horny et al., "Degradation of thermal transport properties in fine-grained isotropic graphite exposed to swift heavy ion beams," Acta Materialia, vol. 184, pp. 187-198, 2020.

[27] W. C. Oliver and G. M. Pharr, "An improved technique for determining hardness and elastic modulus using load and displacement sensing indentation experiments," Journal of Materials Research, vol. 7, no. 6, pp. 1564-1583, 1992.

[28] W. C. Oliver and G. M. Pharr, "Measurement of hardness and elastic modulus by instrumented indentation: advances in understanding and refinements to methodology," Journal of Materials Research, vol. 19, no. 1, pp. 3-20, 2004.

[29] M. Tomut, B. Achenbach, K. H. Behr et al., "Experimental investigations on heavy-ion induced radiation damage of graphite for the super-FRS target and beam catchers," GSI Scientific Report, vol. 2007, p. 69, 2008.

[30] F. Pellemoine, M. Avilov, M. Bender et al., "Study on structural recovery of graphite irradiated with swift heavy ions at high temperature," Nuclear Instruments and Methods in Physics Research Section B: Beam Interactions with Materials and Atoms, vol. 365, pp. 522-524, 2015.

[31] HTW, "SIGRADUR G data sheet," Available from: http:// www.wiyi.com.tw/Download/PDF/Data\%20.pdf, 2020.

[32] A. Leissa, Vibration of Plates, NASA SP-160, Columbus, $\mathrm{OH}$, USA, 1969.

[33] S.-Y. Lin, "Coupled vibration and natural frequency analysis of isotropic cylinders or disks of finite dimensions," Journal of Sound and Vibration, vol. 185, no. 2, pp. 193-199, 1995.

[34] C. Karthik, J. Kane, D. P. Butt, W. E. Windes, and R. Ubic, "In situ transmission electron microscopy of electron-beam induced damage process in nuclear grade graphite," Journal of Nuclear Materials, vol. 412, no. 3, pp. 321-326, 2011.

[35] R. E. Stoller, M. B. Toloczko, G. S. Was, A. G. Certain, S. Dwaraknath, and F. A. Garner, "On the use of SRIM for computing radiation damage exposure," Nuclear Instruments and Methods in Physics Research Section B: Beam Interactions with Materials and Atoms, vol. 310, pp. 75-80, 2013.

[36] A. Bertarelli, "Beam-induced damage mechanisms and their calculation," CERN Yellow Reports, vol. 2, p. 159, 2016.

[37] A. Bertarelli, V. Boccone, F. Carra, and F. Cerutti, "Limits for beam induced damage: reckless or too cautious?" in Proceedings of the Chamonix 2011 workshop on LHC Performance, pp. 183-188, Chamonix, France, January 2011.

[38] C. Karthik, J. Kane, D. P. Butt, W. E. Windes, and R. Ubic, "Neutron irradiation induced microstructural changes in NBG-18 and IG-110 nuclear graphites," Carbon, vol. 86, pp. 124-131, 2015.

[39] H. M. Freeman, A. N. Jones, M. B. Ward et al., "On the nature of cracks and voids in nuclear graphite," Carbon, vol. 103, pp. 45-55, 2016. 